\title{
Mathematical Modelling of Sterile Insect Technology for Mosquito Control
}

\author{
Regina Jijoho Patinvoh', Alfred Akpoveta Susu' \\ ${ }^{1}$ Department of Chemical \& Polymer Engineering, Lagos State University, Lagos, Nigeria \\ ${ }^{2}$ Department of Chemical Engineering, University of Lagos, Lagos, Nigeria \\ Email: alfredasusu222@hotmail.com
}

Received 28 July 2014; revised 1 September 2014; accepted 11 September 2014

Copyright (C) 2014 by authors and Scientific Research Publishing Inc.

This work is licensed under the Creative Commons Attribution International License (CC BY). http://creativecommons.org/licenses/by/4.0/

(c) (i) Open Access

\begin{abstract}
Reduction of mosquito populations will, at least, reduce substantially the transmission of malaria disease. One potential method of achieving this reduction is the environmentally-friendly population control method known as the Sterile Insect Control (SIT) method. The SIT method has so far not been widely used against insect disease vectors, such as mosquitoes, because of various practical difficulties in rearing, sterilization and distribution of the parasite population. For mosquitoes, male-only release is considered essential since sterile females will bite and so may transmit disease, whereas male mosquitoes do not bite. This work concerns the mathematical modelling of the effectiveness of Sterile Insect Technique for Aedes aegypti mosquitoes, when the female sexual preference is incorporated. We found that for a released value of the sterile male mosquito below 40,000 , the wild mosquito population decreases over time while the sterile male mosquito population increases. Therefore, the transmission of malaria and dengue infection declines because the sterile male mosquitoes dominated the environment. We also found that for a released value of the sterile male mosquito above 40,000 , the wild mosquito population decreases and the sterile male mosquito population decreases as well. Therefore, if the injection of sterile male mosquitoes is large enough, the environment will be rid of mosquitoes over time. The result also shows that if sexual selection is incorporated into a reaction diffusion system, modelling the spread of Aedes aegypti mosquitoes, the Sterile Insect Technique (SIT) will still be a successful control measure.
\end{abstract}

\section{Keywords}

Sterile Insect Technique (SIT), Aedes aegypti Mosquitoes, Environment, Malaria Control, Computer Simulation

\section{Introduction}

Malaria is a major life-threatening vector-borne disease transmitted through mosquitoes. The disease got its 
name from bad air (malaria) as it was thought that the disease came from fetid marshes. Later in 1880, it was discovered that the real cause of malaria was Plasmodium [1] $a$ single cell parasite which can only be transmitted from one person to another by the bite of female Anopheles mosquito. The male Anopheles mosquitoes are not involved in disease transmission as they don't require blood to nurture eggs as their female counterparts do [2].

The disease is more prevalent in the tropical and sub-tropical regions of the world and causes more than 300 million acute illnesses and at least one million deaths annually [2]. People living in the world's poorest countries with a total population of approximately $40 \%$ of the world's population, are at a high risk of the malaria ailment. According to the World Malaria Report by World Health Organization (2005), 90\% of deaths caused by malaria take place in Africa, primarily among young children, pregnant women and their unborn children. A child in Africa dies every 30 seconds because of malaria and those who survive the severe episode of malaria might suffer from learning impairments or brain damage. There are no available vaccines yet to prevent infection by the malaria virus and the avoidance of mosquito bites is the most effective protective measures against infection.

In vector borne disease control strategy, it is important to include the mating behavior of the mosquito, which is an aspect of mosquito biology that is not fully understood [3]. The buzz of a flying female mosquito acts as a mating signal for attracting males [4]. Mate assessment interactions in swarming insects occur when these insects enter swarms and this happens very quickly in flight [3]. In mosquito mating swarms, it is important to converge quickly to a mate harmonic signal before others; this is to ensure that a desirable mate locks faster into its signal, than the signal from a swarming competitor [5]. This is an indication that harmonic convergence may be used in mate assessment. The female mosquito has the ability to react to variation in flight tone, which further suggests that this signal may be used to assess the males during precopulatory interactions [6]. Single females fly into the swarm and are detected by their lower wing-beat frequency [7] [8]. It has been reported that several males may arrive near the female, and the female departs with one of the males from the swarm in copula. Also, larger males were more successful in mating than smaller ones [9] [10]. Thus there is evidence that sexual selection operates when these insects enter the swarms, and hence should be incorporated into any reasonable model.

The Sterile Insect Technology has been tried in a number of scenarios, as an attempt to combat malaria and dengue with little success. Many of the earlier approaches were based on ordinary differential equations. They made several assumptions such as 1:1 sex ratio [11], addressing fraction of the population as either infected or uninfected and many more. [12] proposed two mathematical model and proved theoretically and computationally that the control of wild female mosquitoes is effective provided that the number of sterile male mosquitoes released is above the threshold number; the study also exposes the fact that the success of SIT depends on the entomological parameters of the wild anopheles mosquito as well as on parameters released to the sterile males. In this study, we observed the effectiveness of the Sterile Insect Technology for Aedes aegypti mosquitoes when the female sexual preference for wild males over sterile males is incorporated and the computer simulation result shows that if sexual selection is incorporated into a reaction diffusion system, modelling the spread of Aedes aegypti mosquitoes, the sterile insect technology can still be a successful control measure if the injection of sterile males is large enough for a sufficient period.

The objective of this study was to develop a computer program for solving the model equations developed by [13], the dynamics of a PDE model for Aedes aegypti mosquito incorporating female sexual preference and computer simulation to estimate the effectiveness of the Sterile Insect Technology for Aedes aegypti mosquitoes when the female sexual preference for wild males over sterile males is incorporated.

\section{Methodology}

\subsection{Model for Control of Wild Mosquito Population via the Sterile Insect Technology}

PDE model for Aedes aegypti mosquito incorporating female sexual preference is proposed by Parshad and Agusto (2011).

This model is an extension of the model in [14] to include both spatial spread, and preferential selection of the female mosquito for the wild males, against modified sterile males. The mosquito population is divided into male and female classes. The female classes are further divided into immature and adult depending on the insect sexual preference.

The per capita mating rates of an unmating female with a wild male mosquito are given by: 


$$
\begin{array}{r}
\frac{\beta_{I} M}{M+M_{S}} \\
\text { and } \frac{\beta_{P} M}{M+M_{S}}
\end{array}
$$

where: $\beta_{P}<\beta_{I}$

The per capita mating rate of a female with a sterile male is given by:

$$
\frac{\beta_{S} M}{M+M_{S}}
$$

where: $\beta_{S}=p q \beta$ and $0 \leq p ; q \leq 1$

The model is described in Equation (4) and the description of the variables and parameters of the model are shown in Table 1

$$
\begin{aligned}
\frac{\partial A_{I}}{\partial t} & =\Delta A_{I}+\varnothing\left(1-\frac{A_{I}+A_{P}}{C}\right) F_{I}-\left(\gamma+\mu_{A}+\delta\right) A_{I}, \\
\frac{\partial A_{P}}{\partial t} & =\Delta A_{P}+\varnothing\left(1-\frac{A_{P}+A_{I}}{C}\right) F_{P}-\left(\gamma+\mu_{A}\right) A_{P}+\delta A_{I}, \\
\frac{\partial I}{\partial t} & =\Delta I+r \gamma A_{I}-\frac{\beta_{I} M I}{M+M_{S}}-\frac{\beta_{S} M_{S} I}{M+M_{S}}-\mu I, \\
\frac{\partial P}{\partial t} & =\Delta P+r \gamma A_{P}-\frac{\beta_{P} M P}{M+M_{S}}-\mu P, \\
\frac{\partial F_{I}}{\partial t} & =\Delta F_{I}+\frac{\beta_{I} M I}{M+M_{S}}-\mu_{F} F_{I}, \\
\frac{\partial F_{P}}{\partial t} & =\Delta F_{P}+\frac{\beta_{P} M P}{M+M_{S}}-\mu_{F} F_{P}, \\
\frac{\partial M}{\partial t} & =\Delta M+(1-r) \gamma\left(A_{I}+A_{P}\right)-\mu_{M} M, \\
\frac{\partial M_{S}}{\partial t} & =\Delta M_{S}+\alpha-\mu_{M_{S}} M_{S} . \\
\nabla A_{I} \cdot n=\nabla A_{P} \cdot n & =\nabla I \cdot n=\nabla P \cdot n=\nabla F_{I} \cdot n=\nabla F_{P} \cdot n=\nabla M \cdot n=\nabla M_{s} \cdot n=0
\end{aligned}
$$

On $\partial \Omega$ we also impose suitable initial conditions

$$
\begin{aligned}
& A_{I}(x, 0)=A_{I 0}, \quad A_{P}(x, 0)=A_{P 0}, \quad I(x, 0)=I_{0}, \quad P(x, 0)=P_{0}, \\
& F_{I}(x, 0)=F_{I 0}, \quad F_{P}(x, 0)=F_{P 0}, \quad M(x, 0)=M_{0}, \quad M_{S}(x, 0)=M_{S 0} .
\end{aligned}
$$

Assumptions made by Parshad and Agusto (2011):

- A female mosquito mates once in its life, and oviposit its eggs in different places during its entire life [15].

- The population of females with sexual preference is maintained by mutation from female without sexual preference at the rate $\delta$ since the mating rate of the wild females with sexual preference is lower than mating rate of females without sexual preference.

- The females with preference do not mate with the sterile male (even in small probability). This is to incorporate selection in the model, and helps to clearly differentiate between the classes I and P. Note, there is no conclusive evidence, that sexual selection exists in mosquito mating, however there are a number of studies, [3] [6]-[9] that suggest, this might be so.

- We assume what is known as a mutation-selection balance, [4]. Essentially, we assume that females that display preference in mate selection have a lower mating rate overall (e.g. due to time wasted searching for a male). In the absence of a high number of fit males, these females are at a fitness disadvantage, because they do not reproduce as quickly. As a result, over time, they would be driven to extinction by competitive exclu- 
sion with the females without preference. Thus preference can be thought of as a deleterious allele. In many population biology models, deleterious alleles are assumed maintained in the population by mutation. The deleterious alleles keep appearing as a result of mutation, but because they are selected against, they are only maintained at low levels.

\subsection{Model Simulation}

In simulating, model Equation (4), the parameter values in Table 2 and the assumed initial values in Table 3 were used. The computer program and algorithm for the simulation is shown in appendix 1 and 2 while the flowchart is shown in Figure 1.

$p=$ ability of dispersion, $q=$ percentage of reduction of mating capacity and $\alpha=$ release rate of sterile male mosquitoes Values of $p$ are sensitive to the environment into which the genetic male mosquito is being released [18] [19].

Table 1. Description of the variables and parameters of the model.

\begin{tabular}{|c|c|}
\hline Variable & Description \\
\hline$A(t)$ & Immature phase of insect \\
\hline$I(t)$ & Single females with male preference \\
\hline$P(t)$ & Single females with wild male preference \\
\hline$F(t)$ & Mating fertilized females \\
\hline$M(t)$ & Wild males \\
\hline$M s(t)$ & Sterile males \\
\hline Parameter & Description \\
\hline$C$ & Carrying capacity related to the amount of available nutrients and space \\
\hline$\varnothing$ & Intrinsic oviposition rate \\
\hline$\beta$ & Mating rate of natural insects \\
\hline$\beta T=q p \beta$ & Mating rate if SIT \\
\hline$q$ & Percentage of reduction of mating capacity \\
\hline$p$ & Ability of dispersion \\
\hline$\gamma$ & Mature rate to adulthood \\
\hline$r \gamma$ & Female mature rate to adulthood \\
\hline$(1-r) \gamma$ & Male mature rate to adulthood \\
\hline$\mu A, \mu, \mu F, \mu M, \mu \mathrm{Ms}$ & Natural mortality rate \\
\hline$\alpha$ & Release rates of sterile male mosquitoes \\
\hline
\end{tabular}

Courtesy: [13].

Table 2. Parameter values used for simulation study [16] [17].

\begin{tabular}{|c|c|c|c|c|c|}
\hline $\begin{array}{l}\mu_{A} \text { (mortality rates of } \\
\text { the immature form) }\end{array}$ & $\begin{array}{l}\mu \text { (mortality rates of } \\
\text { the unmating female) }\end{array}$ & $\begin{array}{l}\mu_{F} \text { (mortality rates of } \\
\text { the fertilized female) }\end{array}$ & $\begin{array}{l}\mu_{M} \text { (mortality rates } \\
\text { of the wild males) }\end{array}$ & $\begin{array}{l}\mu_{M s} \text { (mortality rates } \\
\text { of the sterile males) }\end{array}$ & $\begin{array}{l}\beta_{T} \text { (mating } \\
\text { rates of SIT) }\end{array}$ \\
\hline 0.50 & 0.50 & 0.50 & 0.1 & 0.1 & $q p \beta=0.7$ \\
\hline $\begin{array}{c}\Phi \quad \text { (intrinsic } \\
\text { oviposition rate) }\end{array}$ & $\begin{array}{l}\gamma \quad \text { (mature rate to } \\
\text { adulthood) }\end{array}$ & $\begin{array}{c}r \text { (proportion that } \\
\text { transforms into female) }\end{array}$ & $\begin{array}{l}\beta \text { (mating rate of } \\
\text { natural insect) }\end{array}$ & $\begin{array}{l}C \text { (carrying capacity } \\
\text { related to amounts of } \\
\text { available nutrients }\end{array}$ & $p q$ \\
\hline 5.0 & 0.075 & 0.5 & 1.0 & 600 & 0.7 \\
\hline
\end{tabular}


Table 3. Assumed Initial values used for simulation.

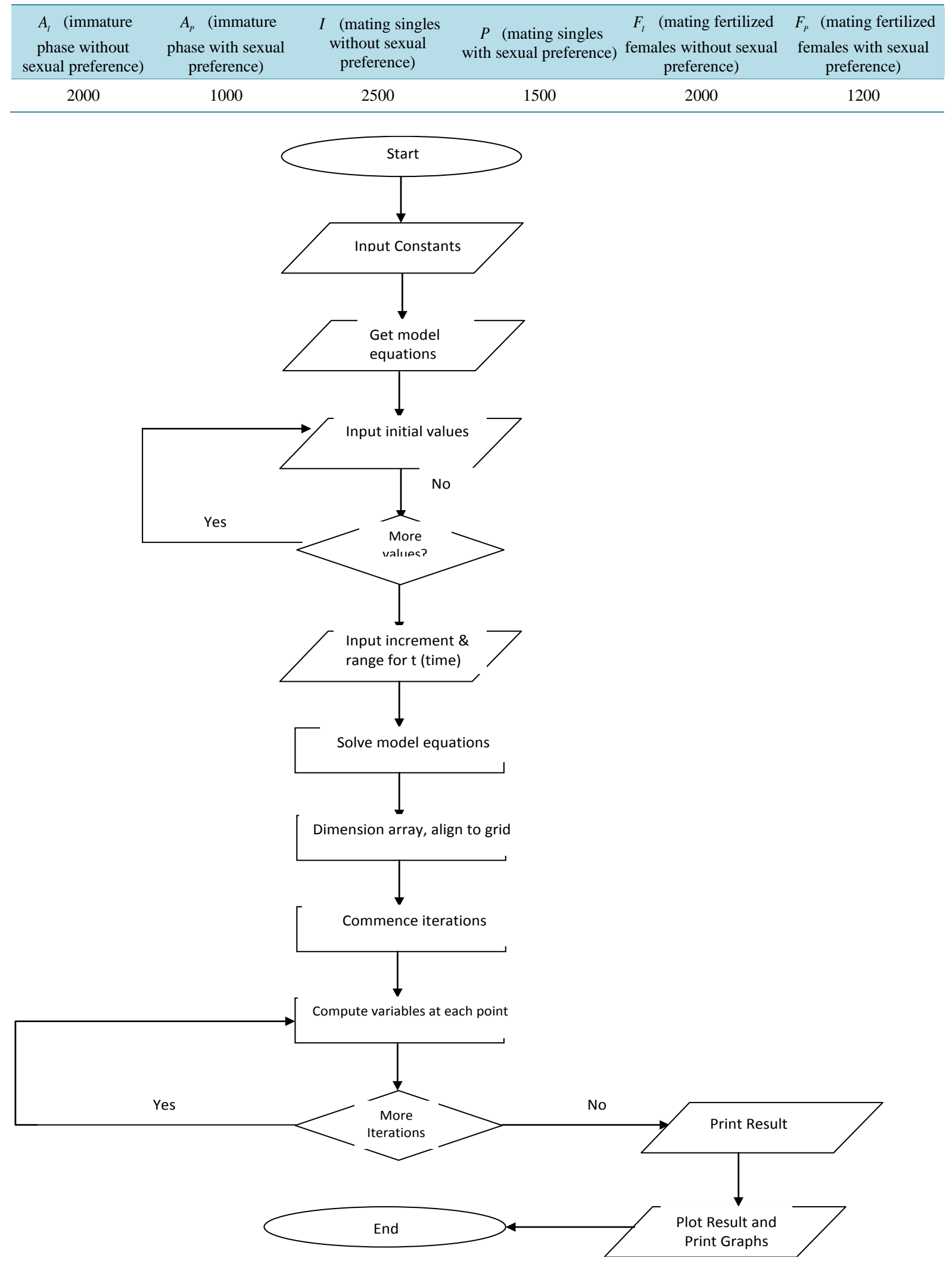

Figure 1. Model simulation flowchart. 


\section{Results}

The computer program for the simulation is presented in Appendix 1.

In the simulation presented on Figures 2-5, we used model Equation (4), the parameter values in Table 2 and assumed initial values as shown in Table 3. The model was solved using MATLAB assuming constant initial values while varying the initial value of released sterile male mosquitoes. For the population control, the ratio of the number of released sterile males to the number of wild males in the local population was 10:1.

Figure 2(a) shows the wild mosquito population dynamics while Figure 2(b) shows the sterile male mosquito population dynamics when the initial released sterile male was 4000. Figure 3(a) and Figure 3(b) show the population dynamics for both the wild mosquito and sterile male when the initial released sterile male was 6000 . Figure 4(a) and Figure 4(b) show the population dynamics for both the wild mosquito and sterile male when the initial released sterile male was 50,000 and Figure 5(a) and Figure 5(b) show the population dynamics for both the wild mosquito and sterile male when the initial released sterile male was 100,000.

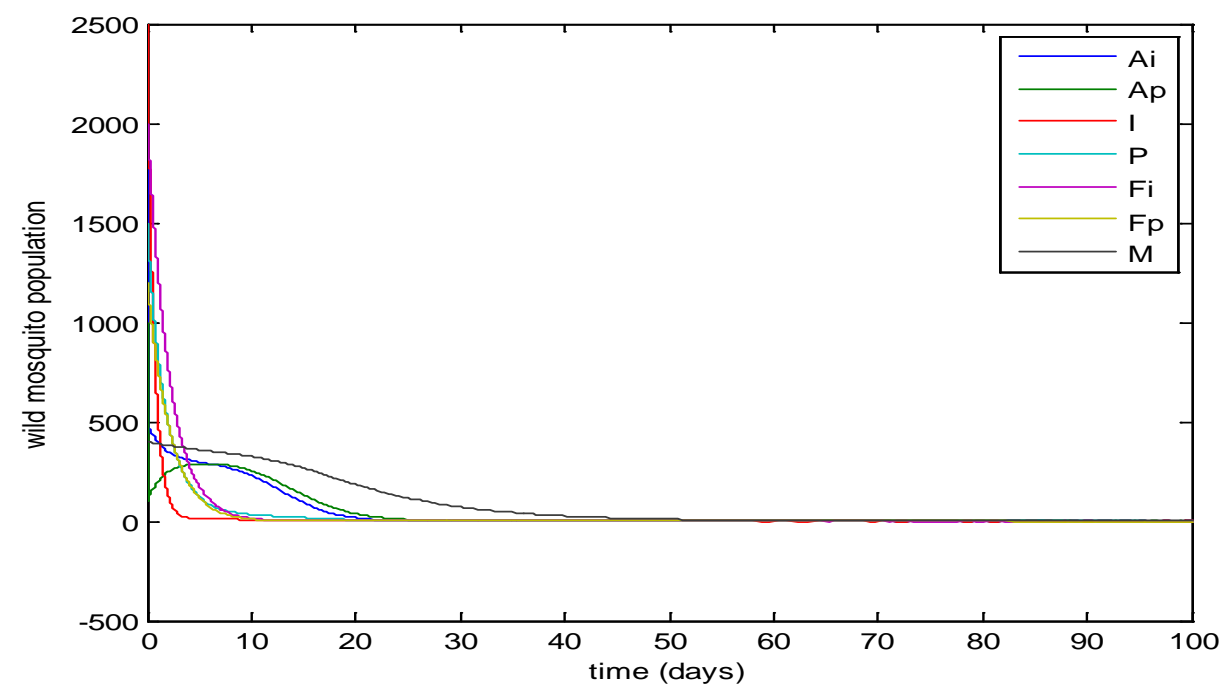

(a)

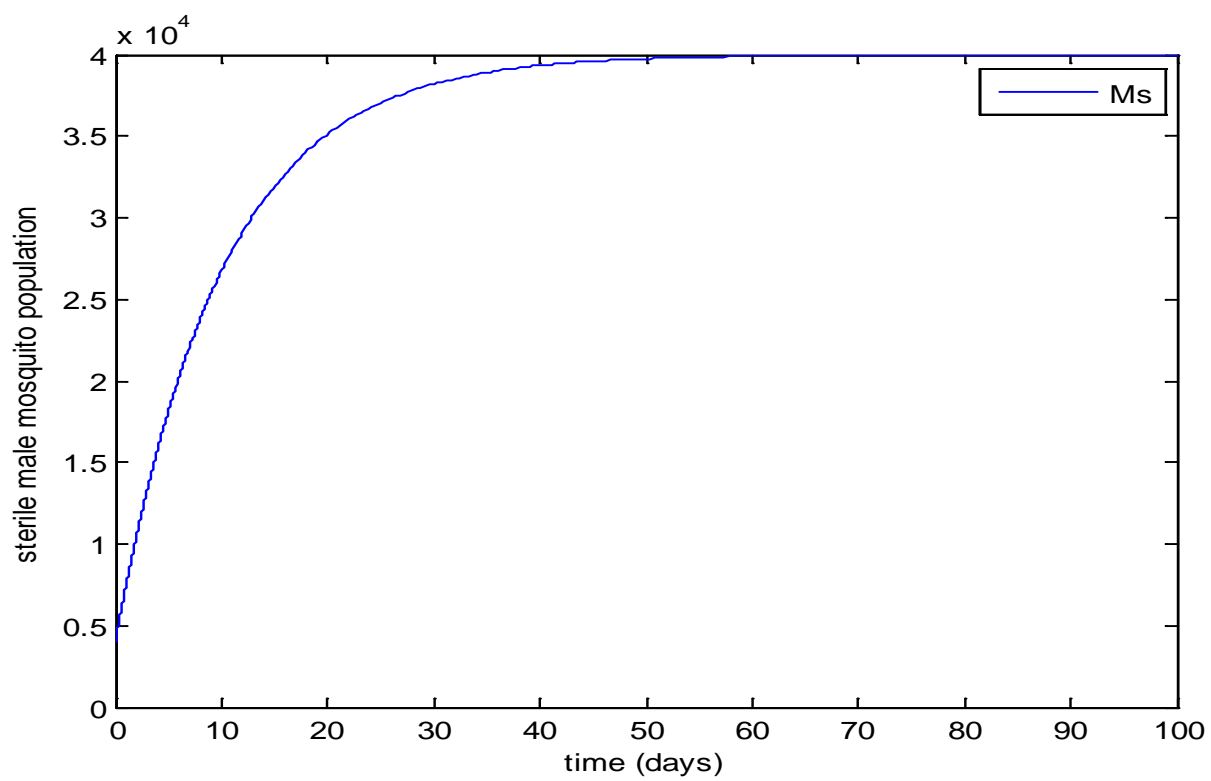

(b)

Figure 2. (a) Dynamics conditions of population under SIT control (Ms = 4000). (b) Dynamics conditions of population under SIT control $(\mathrm{Ms}=4000)$. 


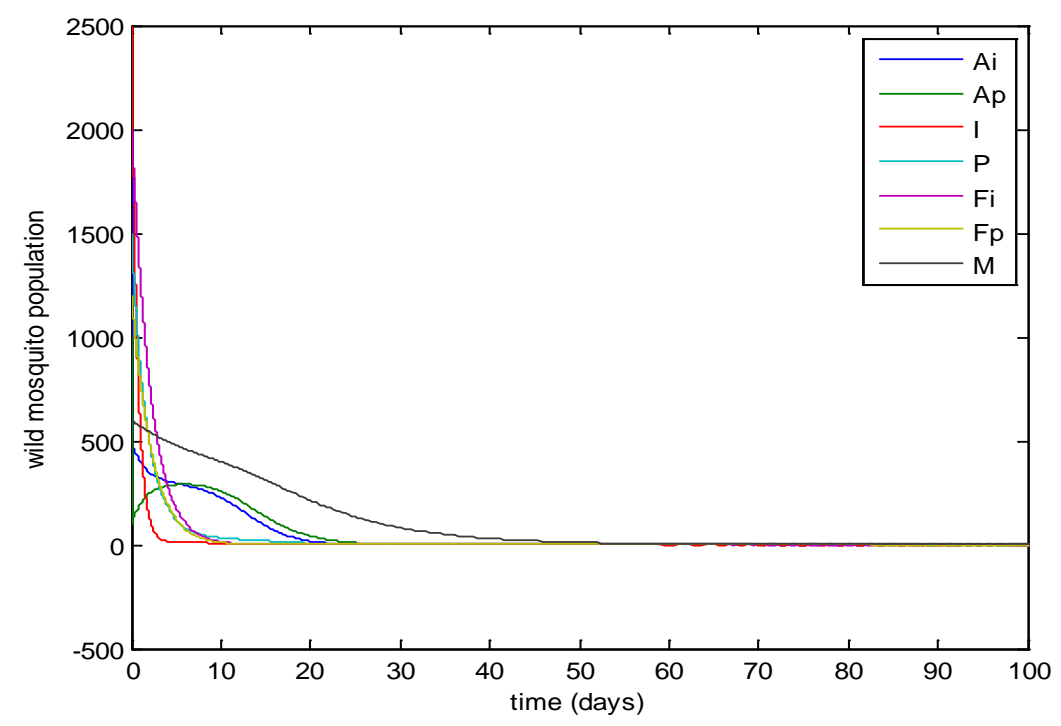

(a)

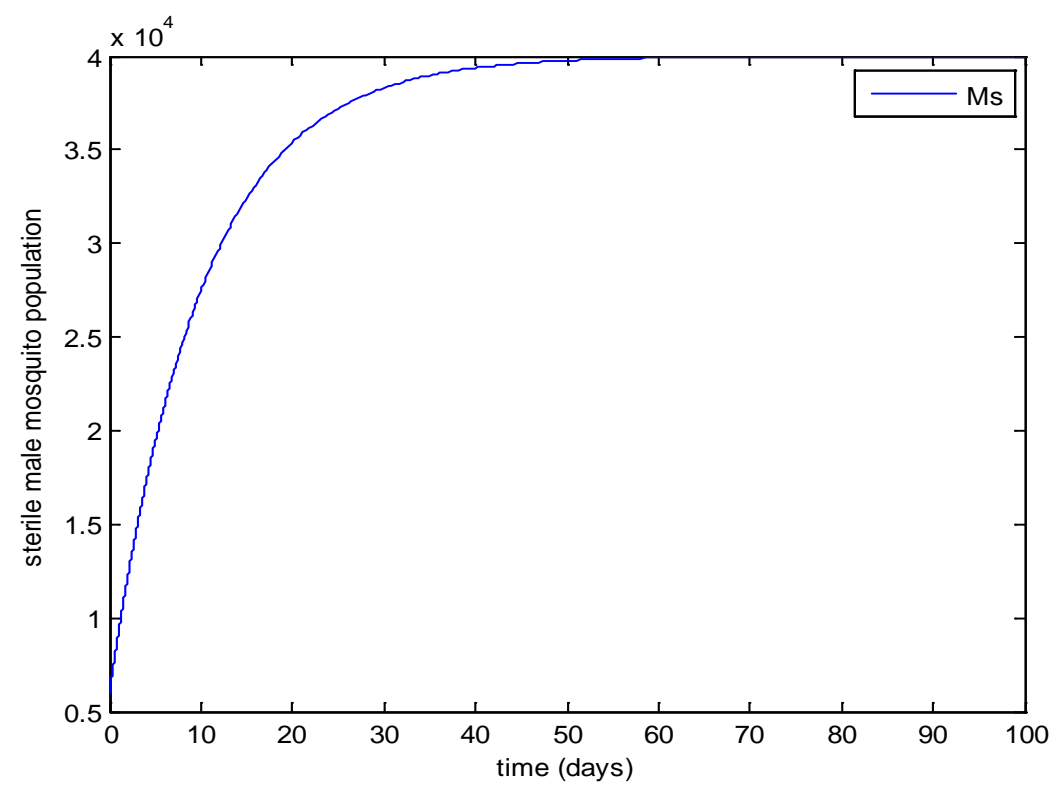

(b)

Figure 3. (a) Dynamics conditions of population under SIT control $(\mathrm{Ms}=6000)$. (b) Dynamics conditions of population under SIT control $(\mathrm{Ms}=6000)$.

\section{Discussion of Results}

The sterile insect technique (SIT) is a biological control which disorders the natural reproductive process of insects; male insects are first made sterile by gamma radiation before releasing them in large numbers into the environment to mate with the native wild insects. Insect populations can be controlled by the release of large numbers of sterile males. Thus, if a female mate with a male that has no sperm or whose sperm was rendered unviable, this female will have fewer or no offspring. When many sterile males are released, the local population tends to decline or become wiped out. For population control, the crucial parameter is the ratio of the number of released sterile males to the number of males in the local population, which ideally should be around 10:1 [20].

As demonstrated on Figure 4(a) and Figure 4(b) for sterile male mosquito released below 40,000, the wild mosquito population decreases while the sterile male mosquito population increases. For mosquitoes, male-only release is considered essential since sterile females will bite and so may transmit disease, whereas male mosque- 


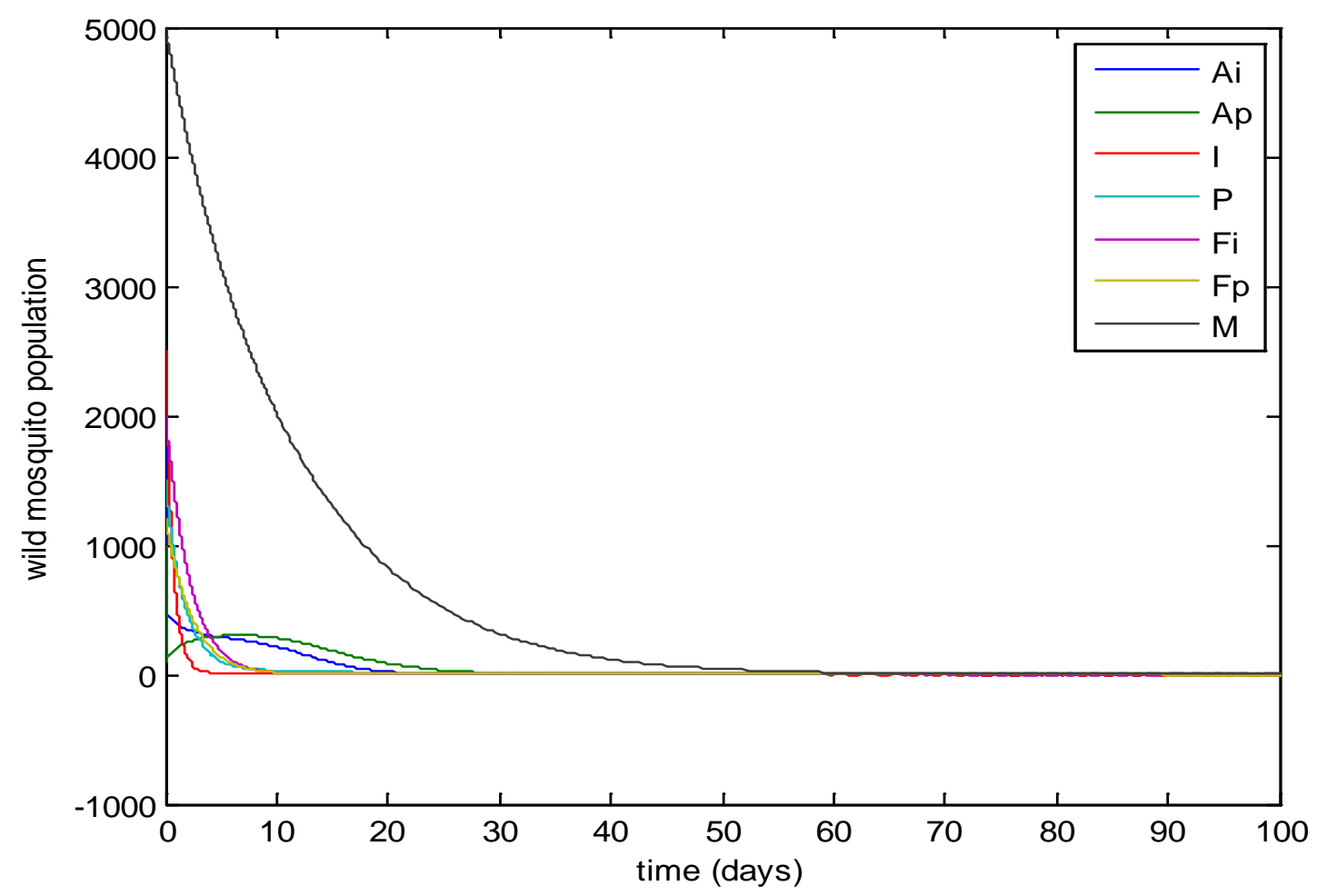

(a)

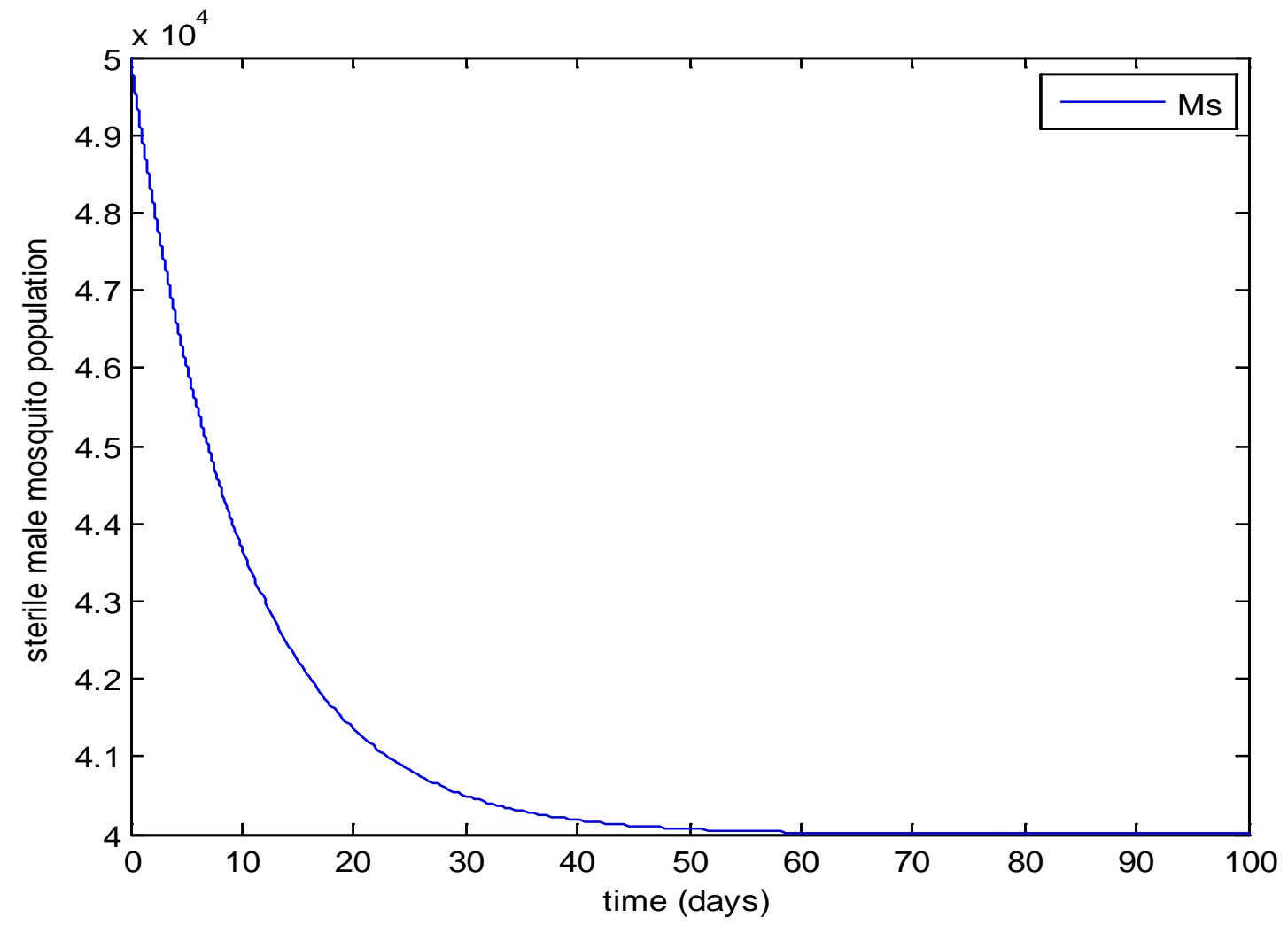

(b)

Figure 4. (a) Dynamics conditions of population under SIT control (Ms = 50,000). (b) Dynamics conditions of population under SIT control $(\mathrm{Ms}=50,000)$. 


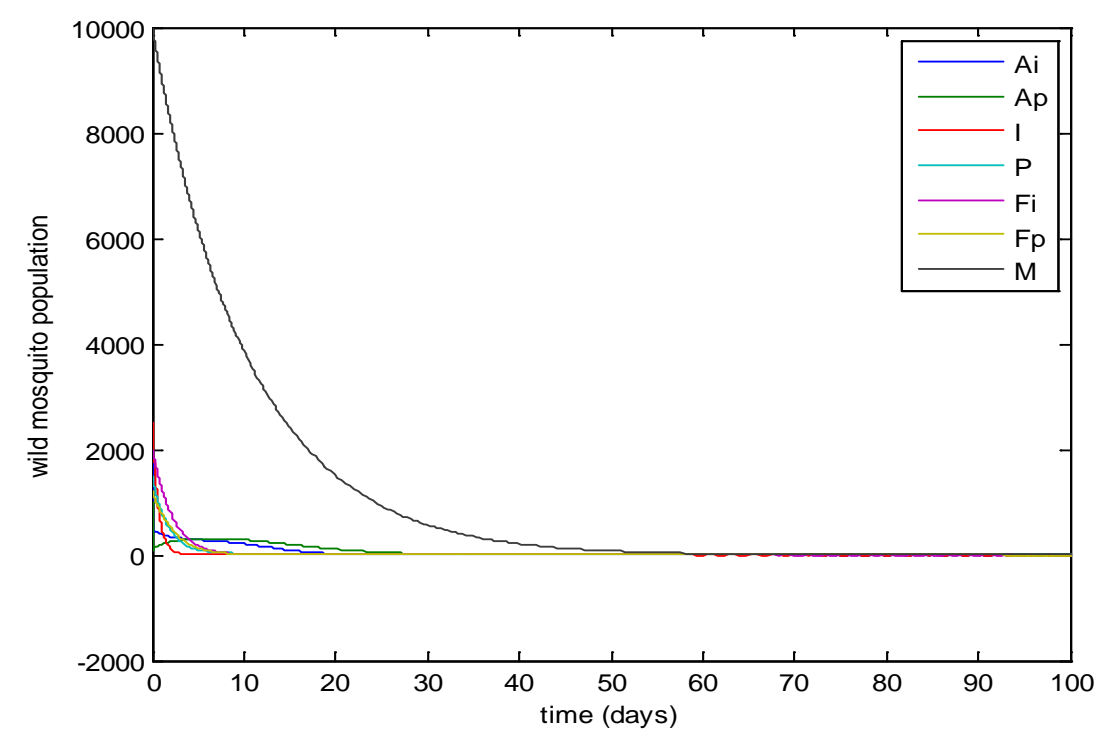

(a)

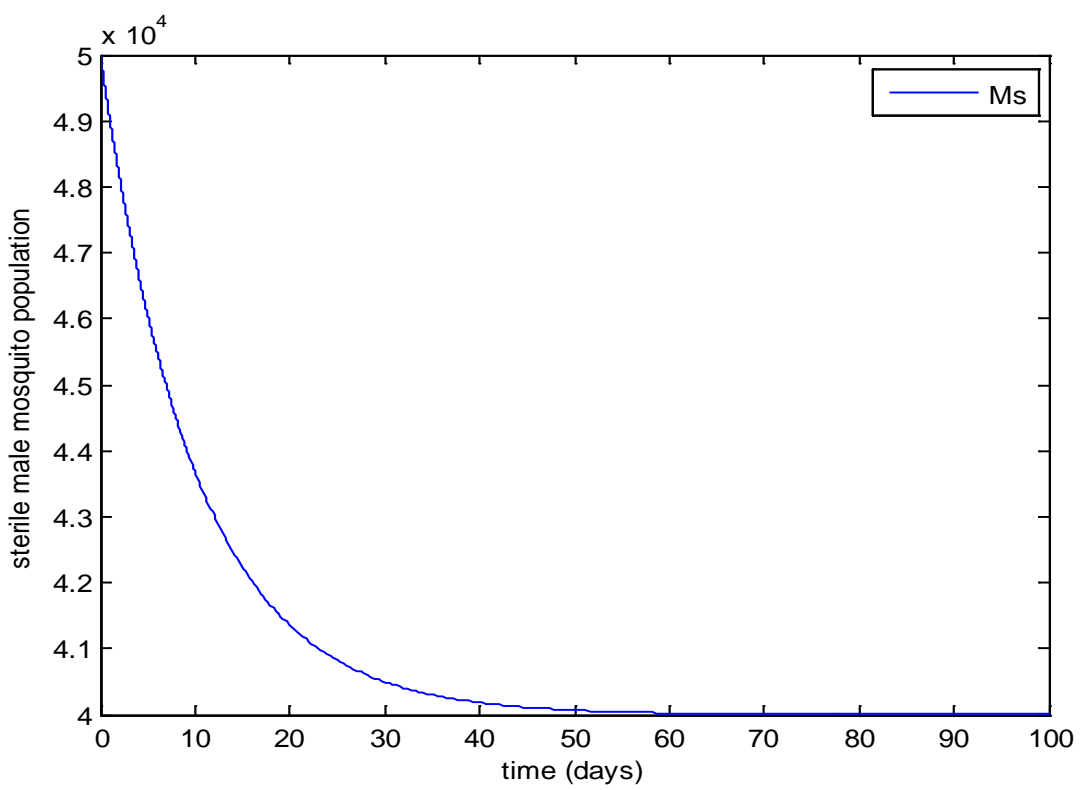

(b)

Figure 5. (a) Dynamics conditions of population under SIT control (Ms = 100,000). (b) Dynamics conditions of population under SIT control (Ms $=100,000)$.

toes do not bite. These released insects compete for mates with wild males; a wild female mating with a released sterile male has no or fewer progeny, so the population tends to decline. This means that the sterile male mosquitoes have dominated the environment. Hence if the mosquito population has not grown yet sufficiently or it has been reduced by some other measure e.g. destroying the breeding places, it can be controlled by release of sterile males.

As shown in Figure 4(a) and Figure 4(b) for sterile male mosquito released above 40,000, the wild mosquito population decreases and the sterile male mosquito population decreases as well. In SIT, some or all of the progeny of released individuals die as a consequence of inheriting one or more dominant lethal mutations, so the population tends to decline. For population control purposes, the only timing requirement for the lethal system is that death occurs before reproductive maturity.

This implies that if sufficient sterile male mosquitoes are released for a sufficient period, the wild mosquito 
population decreases to zero and so the environment is dominated by the sterile male mosquitoes and with time these sterile male mosquitoes start dying since there is no reproduction. Therefore, if the injection of sterile male mosquitoes is large enough the environment will be eradicated completely of mosquitoes over time.

Essentially our results show that if sexual selection is incorporated into a reaction diffusion system, modeling the spread of Aedes aegypti mosquitoes, the sterile insect technique can still be a successful control measure, if the injection of sterile males is large enough.

\section{Conclusions}

From the simulation, it was revealed that the success of SIT depends on the parameters of the wild mosquitoes as well as on parameters related to the sterile male mosquitoes.

We established that for a released value of the sterile male mosquito below 40,000 , there was reduction in the wild mosquito population and domination of the sterile male mosquitoes in the environment. For a released value of the sterile male mosquito above 40,000, there was eradication of mosquitoes in the environment over time as the sterile male mosquito population decreases. The result shows that if sexual selection is incorporated into a reaction diffusion system, modelling the spread of Aedes aegypti mosquitoes, the sterile insect technology can still be a successful control measure if the injection of sterile males is large enough. Therefore, if sufficient sterile insects are released for a sufficient period, the target population will be controlled or even locally eradicated.

\section{Acknowledgements}

This project was supported by Lagos State Government (Local Scholarship Award). The authors would like to thank Mogbojuri Babatunde (Lead software developer/system engineer) at Global Accelerex Ltd. who was there in the course of this project to provide answers to every professional question.

\section{References}

[1] Roll Back Malaria (2001) Country Strategies and Resource Requirements. WHO/CDS/RBM/2001.34

[2] Ribeiro, J.M.C. (1987) Role of Saliva in Blood-Feeding by Arthropods. Annual Review of Entomology, 32, 463-478. http://dx.doi.org/10.1146/annurev.en.32.010187.002335

[3] Cator, L.J, Arthur, B.J., Harrington, L.C. and Hoy, R.R. (2009) Harmonic Convergence in the Love Songs of the Dengue Vector. Mosquito. Science, 323, 1077-1079.

[4] Crow, J.F. (1986) Basic Concepts in Population, Quantitative, and Evolutionary Genetics. W.H. Freeman, New York, 273.

[5] Lee, N., Elias, D.O. and Mason, A.C. (2009) A Precedence Effect Resolves Phantom Sound Source Illusions in the Parasitoid Fly Ormia ochracea. Proceedings of the National Academy of Sciences of the United States of America, 106, 6357-6362. http://dx.doi.org/10.1073/pnas.0809886106

[6] Cator, L.J., NgHabi, K.R., Hoy, R.R. and Harrington, L.C. (2010) Sizing up a Mate: Variation in Production and Response to Acoustic Signals in Anopheles gambiae. Behavioral Ecology, 21, 1033-1039. http://dx.doi.org/10.1093/beheco/arq087

[7] Belton, P, (1994) Attraction of Male Mosquitoes to Sound. Journal of the American Mosquito Control Association, 10, 297-301.

[8] Clements, A.N. (1999) The Biology of Mosquitoes. Sensory Reception and Behavior. CABI Publishing Inc., New York.

[9] Yuval, B. and Bouskila, A. (1993) Temporal Dynamics of Mating and Predation in Mosquito Swarms. Oecologia, 85, 65-69.

[10] Yuval, B., Wekesa, J.W. and Washino, R.K. (1993) Effects of Body Size on Swarming Behavior and Mating Success of Male Anopheles Freeborni (Diptera: Culicidae). Journal of Insect Behavior, 6, 333-342. http://dx.doi.org/10.1007/BF01048114

[11] Engelstädter, J. (2010) The Effective Size of Populations Infected with Cytoplasmic Sex-Ratio Distorters. Genetics, 186, 309-320. http://dx.doi.org/10.1534/genetics.110.120014

[12] Anguelov, R., Dumont, Y. and Lubuma, J. (2012) Mathematical Modelling of Sterile Insect Technology for Control of Anopheles Mosquito. Computers and Mathematics with Applications, 64, 374-389. http://dx.doi.org/10.1016/j.camwa.2012.02.068

[13] Parshad, R.D. and Agusto, F.B. (2011) Global Dynamics of a PDE Model for Aedes aegypti Mosquitoe Incorporating 
Female Sexual Preference. Dynamics of Partial Differential Equations, 8, 311-343.

[14] Thomé, R.C.A, Yang, H.M. and Esteva, L. (2010) Optimal Control of Aedes aegypti Mosquitoes by the Sterile Insect Technique and Insecticide. Mathematical Biosciences, 223, 12-23. http://dx.doi.org/10.1016/j.mbs.2009.08.009

[15] Bartlett, A.C. (1990) Insect, Sterility, Insect Genetics, and Insect Control. In: Pimentel, D., Ed., Handbook of Pest Management in Agriculture, CRC Press, Boca Raton, 279-287.

[16] Esteva, L. and Yang, H.M. (2005) Mathematical Model to Assess the Control of Aedes aegypti Mosquitoes by the Sterile Insect Technique. Mathematical Biosciences, 198, 132-147. http://dx.doi.org/10.1016/j.mbs.2005.06.004

[17] Gubler, D.J. (1986) Dengue, the Arboviruses, Epidemiology and Ecology. Vol. 11, Monath, T.P., Ed., p. 213.

[18] Rafikov, M., Bevilacqua, L. and Wyse, A.P.P. (2009) Optimal Control Strategy of Malaria Vector Using Genetically Modified Mosquitoes. Journal of Theoretical Biology, 258, 418-429. http://dx.doi.org/10.1016/j.jtbi.2008.08.006

[19] Takahashi, L.T., Maidana, N.A., Ferreira Jr., W.C., Pulino, P. and Yang, H.M. (2005) Mathematical Models for the Aedes aegypti Dispersal Dynamics: Travelling Waves by Wing and Wind. Bulletin of mathematical Biology, 67, 509528. http://dx.doi.org/10.1016/j.bulm.2004.08.005

[20] Jacob-Lorena, M. Genetic Approaches for Malaria Control. Johns Hopkins School of Public Health, Malaria Research Institute, Dept. Molecular Microbiology and Immunology, Baltimore.

\section{Notations}

$I=\quad$ for the adult form, mating singles without sexual preference

$P=\quad$ for the adult form, mating singles with sexual preference

$A_{I}=\quad$ immature phase of the mosquitoes (eggs, larvae and pupae) from adults without sexual preference

$A_{P}=$ immature phase from adults with sexual preference

$F_{I}=$ mating fertilized females without sexual preference

$F_{P}=$ mating fertilized females with sexual preference

$M=$ wild male mosquito population

$M_{S}=$ sterile insect (sterile male mosquitoes)

$\Phi=\quad$ oviposition rate per female mosquito which is proportional to female density, but it is also regulated by a carrying capacity effect, $C$, which is related to the amount of available nutrients and space

$\gamma=\quad$ the rate at which the aquatic population becomes winged mosquitoes

$r=\quad$ proportion that transforms into female

$1-r=$ proportion that transforms into male

$\mu_{A}=$ mortality rates of the immature form with and without sexual preference

$\mu_{F}=$ mortality rates of the fertilized female with and without sexual preference

$\mu=\quad$ mortality rates of the unmating female with and without sexual preference

$\mu_{M}=$ mortality rates of the wild males

$\mu_{\mathrm{MS}}=$ mortality rates of the sterile males

$\alpha=\quad$ the rate at which sterile males are released and sprayed

$p=\quad$ the effectiveness of sterile male introduction regarded to the spatial distribution of female insects

$q=$ physiological modifications induced by the sterilization technique 


\section{Appendix 1}

The computer Program for the simulation of a PDE model developed by Parshad and Agusto 2011 for Aedes aegypti mosquito using SIT when the female sexual preference is incorporated. Function $\mathrm{mc}=$ mosquito control $(t, x) n=8$; \% n represents the number of equations or variables $\mathrm{mc}=\operatorname{zeros}(n, 1)$;

\section{Part A}

This section of the code declares all constants

mua $=0.5 ; \%$ mortality rates of the immature form with and without sexual preference $\mathrm{mu}=0.5 ; \%$ mortality rates of the unmating female with and without sexual preference muf $=0.5 ; \%$ mortality rates of the fertilized female with and without sexual preference mum $=0.1 ; \%$ mortality rates of the wild males mums $=0.1 ; \%$ mortality rates of the sterile males

$\mathrm{Bi}=1.0$;

del $=0.12$;

$\mathrm{Bp}=1.0$

$\mathrm{Bs}=1.0$

phi $=50 ; \%$ oviposition rate per female mosquito

gamma $=0.1 ; \%$ the rate at which the aquatic population becomes winged mosquitoes

$r=0.5$; \% proportion that transforms into female

$C=600 ; \%$ carrying capacity effect

alpha $=4000$; \%rate at which sterile males are released and sprayed

This section of the code solves the system of PDE's

$\%$ the following definitions apply

$\% x(1)=A i$-Immature phase of the mosquitoes

$\% x(2)=A p$-immature [phase from adults with sexual preference

$\% x(3)=I$-for the adult form, mating singles without sexual preference

$\% x(4)=P$-for the adult form, mating singles with sexual preference

$\% x(5)=F i$-mating fertilized females without sexual preference

$\% x(6)=F p$-mating fertilized females with sexual preference

$\% x(7)=M$-wild male mosquito population

$\% x(8)=$ Ms -sterile insect (sterile male mosquitoes)

$\mathrm{mc}(1)=\operatorname{phi} \times(1-(x(1)+x(2)) / C) \times x(5)-($ gamma + mua $+\operatorname{del}) \times x(1)$;

$\mathrm{mc}(2)=\operatorname{phi} \times(1-(x(2)+x(1)) / \mathrm{C}) \times x(6)-($ gamma + mua $) \times x(2)+\operatorname{del} \times x(1)$;

$\mathrm{mc}(3)=r \times \operatorname{gamma} \times x(1)-(\operatorname{Bi} \times x(7) \times x(3) /(x(7)+x(8)))-(\operatorname{Bs} \times x(8) \times x(3) /(x(7)+x(8)))-\operatorname{mu} \times x(3)$;

$\mathrm{mc}(4)=r \times \operatorname{gamma} \times x(2)-(\operatorname{Bp} \times x(7) \times x(4) /(x(7)+x(8)))-\operatorname{mu} \times x(4)$;

$\mathrm{mc}(5)=(\mathrm{Bi} \times x(7) \times x(3) /(x(7)+x(8)))-$ muf $\times x(5)$;

$\mathrm{mc}(6)=(\operatorname{Bp} \times x(7) \times x(4) /(x(7)+x(8)))-\operatorname{muf} \times x(6)$;

$\mathrm{mc}(7)=(1-r) \times \operatorname{gamma} \times(x(1)+x(2))-\operatorname{mum} \times x(7)$;

$\mathrm{mc}(8)=$ alpha $-(\operatorname{mums} \times x(8))$;

\section{Part B}

This section allows user to specify initial value of variables for the system of PDE's

$A i 0=$ input ("Enter a value for $A i(0)=$ ");

$A p 0=$ input ("Enter a value for $A p(0)=$ ");

$I 0=$ input ("Enter a value for $I(0)=$ ");

$P 0=$ input ("Enter a value for $P(0)=$ ");

$F i 0=$ input ("Enter a value for $F i(0)=$ ");

$F p 0=$ input ("Enter a value for $F p(0)=$ ");

$M 0=$ input ("Enter a value for $M(0) \quad=\quad$ ");

Ms0 = input ("Enter a value for Ms(0) = ");

This section solves the system of PDE's

$x 0=[$ Ai0 Ap0 I0 P0 Fi0 Fp0 M0 Ms0]; 
$[t, x]=$ ode45(@mosquitocontrol, [0 100], x0)

This section generates the graph of the system of PDE's

Plot $(t, x(:, 1), t, x(:, 2), t, x(:, 3), t, x(:, 4), t, x(:, 5), t, x(:, 6), t, x(:, 7), t, x(:, 8))$;

xlabel ('time (days)');

ylabel ("population");

legend ('Ai', 'Ap', 'T', 'P', 'Fi', 'Fp', 'M', 'Ms')

title (“Graph showing population distribution of mosquitoes”);

figure;

plot $(t, x(:, 1), t, x(:, 2), t, x(:, 3), t, x(:, 4), t, x(:, 5), t, x(:, 6), t, x(:, 7))$;

xlabel (“time (days)");

ylabel ("population");

legend ('Ai','Ap','I','P','Fi','Fp','M')

title ("Graph showing population distribution of mosquitoes”);

figure;

plot $(t, x(:, 8))$;

xlabel ("time (days)");

ylabel ("population");

legend ("Ms")

figure;

plot $(t, x(:, 7))$;

xlabel ("time (days)”);

ylabel ("population");

legend (" $M$ ")

figure;

plot $(t, x(:, 6))$;

xlabel ("time (days)");

ylabel ("population");

legend (“Fp")

figure;

plot $(t, x(:, 5))$;

xlabel ("time (days)");

ylabel ("population”);

legend ("Fi")

figure;

plot $(t, x(:, 4))$;

xlabel ("time (days)");

ylabel (“population”);

legend ("P")

figure;

plot $(t, x(:, 3))$;

xlabel ("time (days)");

ylabel ("population”);

legend ("I")

figure;

plot $(t, x(:, 2))$;

xlabel ("time (days)”);

ylabel ("population");

legend (“Ap")

figure;

plot $(t, x(:, 1))$;

xlabel ("time (days)");

ylabel ("population”);

legend (“Ai") 


\section{Appendix 2}

Algorithm for Mosquito control model

1) Define model parameters and variables

2) Input values of constants $\alpha, \beta_{I}, \beta_{P}, \beta_{S}, \varnothing, \gamma, \mu_{A}, \mu, \mu_{F}, \mu_{M}, \mu_{\mathrm{Ms}}, C, r, \delta$

3) Specify model equation

4) Input initial values of variables $A, A_{P}, I, P, F_{I}, F_{P}, M, M_{S}$

5) Determine solution method for system of equations (4th order Runge-Kutta method)

6) Specify step increment and simulation range for independent variable (time)

7) Solve model equations using solution method specified above

8) Dimension array and align to grid

9) Start iterations

10) Compute variables at each point

11) Continue loop until last point

12) Print result

13) Plot result and print graphs

14) End simulation 
Scientific Research Publishing (SCIRP) is one of the largest Open Access journal publishers. It is currently publishing more than 200 open access, online, peer-reviewed journals covering a wide range of academic disciplines. SCIRP serves the worldwide academic communities and contributes to the progress and application of science with its publication.

Other selected journals from SCIRP are listed as below. Submit your manuscript to us via either submit@scirp.org or Online Submission Portal.
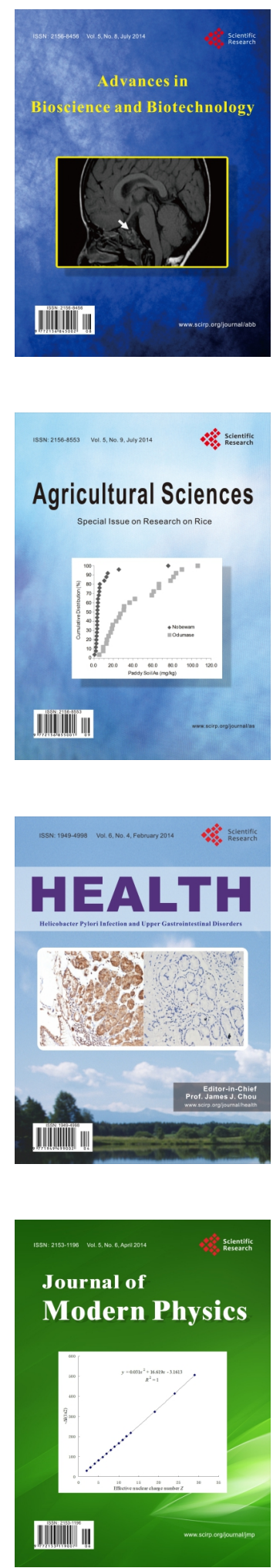
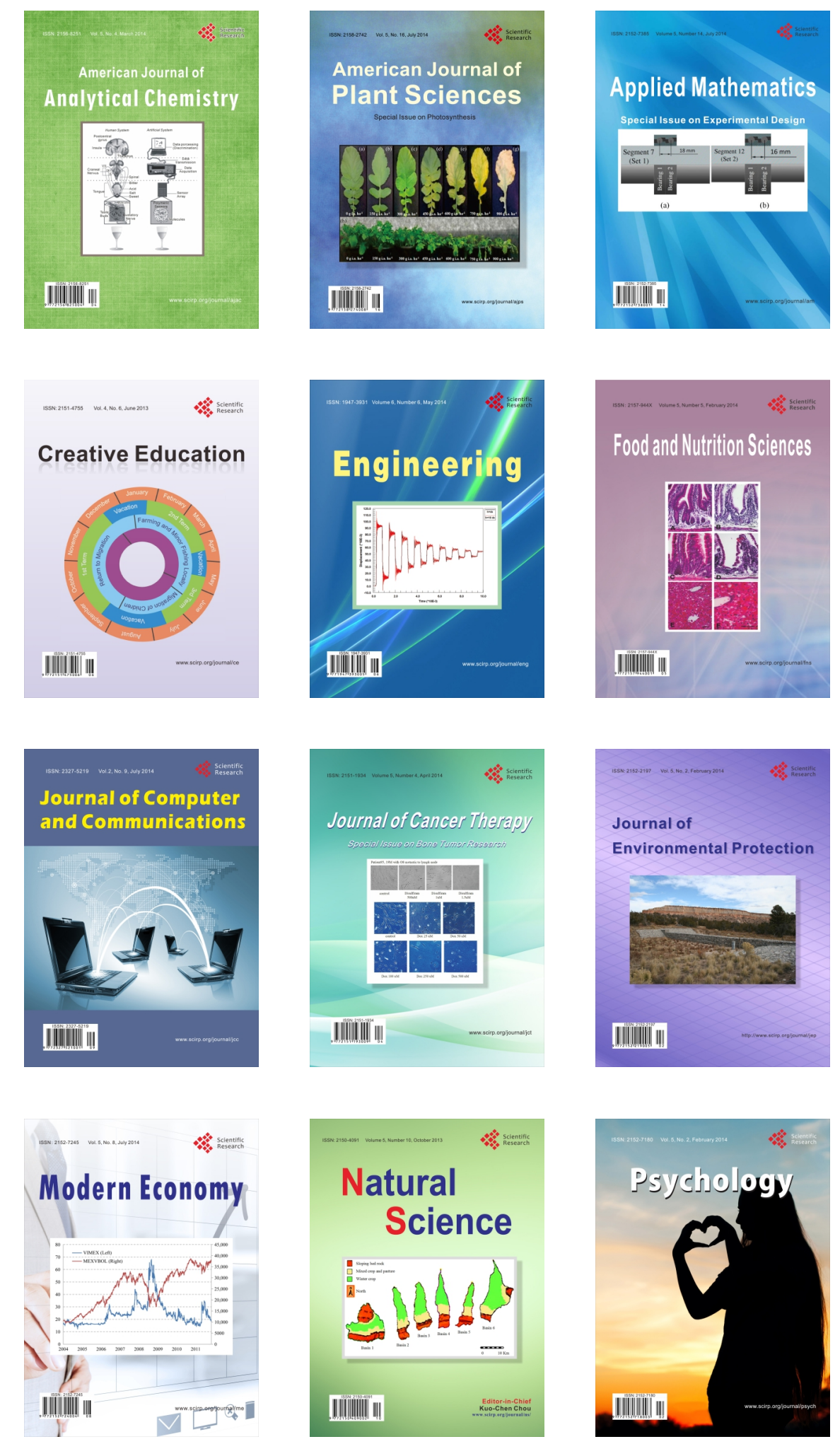